\title{
Diaminodiphenyl Sulfone-Induced Hemolytic Anemia and Alopecia in a Case of Linear IgA Bullous Dermatosis
}

\author{
Eijiro Akasaka ${ }^{a}$ Sato-Jin Kayo ${ }^{a}$ Hajime Nakano ${ }^{a}$ Norito Ishii ${ }^{b}$ \\ Takashi Hashimoto $^{c}$ Daisuke Sawamura ${ }^{a}$ \\ ${ }^{a}$ Department of Dermatology, Hirosaki University Graduate School of Medicine, Hirosaki, and \\ ${ }^{b}$ Department of Dermatology, Kurume University School of Medicine, and ' Kurume University \\ Institute of Cutaneous Cell Biology, Kurume, Japan
}

\section{Key Words}

Linear immunoglobulin A bullous dermatosis · Diaminodiphenyl sulfone · Anemia · Hair loss

\begin{abstract}
Linear immunoglobulin A (IgA) bullous dermatosis (LABD) is an autoimmune mucocutaneous disease characterized by subepidermal blistering induced by IgA autoantibodies against several autoantigens in the basal membranous zone of the skin and mucosal tissue. Although diaminodiphenyl sulfone (DDS), also known as dapsone, is generally recognized as the firstline therapy for $L A B D, D D S$ can induce several severe side effects. We present a Japanese case of LABD with DDS-induced hemolytic anemia and alopecia. In the present case, the DDS-induced hemolytic anemia and hair loss made the DDS monotherapy difficult. When DDS is used in LABD patients with iron deficiency anemia (IDA), hemolytic anemia is concealed by IDA. It is thus necessary to carefully and frequently examine the laboratory data to find the signs of DDS-induced hemolytic anemia. Even though there is no literature on DDSinduced alopecia, alopecia was reported as one of the side effects of DDS in an FDA report, and, in our case, hair loss was improved after reducing its dosage. We have to recognize that alopecia is one of the side effects of DDS and that careful management is needed in order not to overlook the adverse side effects of DDS when treating LABD patients.
\end{abstract}


Case Reports in
Dermatology

\begin{tabular}{l|l}
\hline \multicolumn{2}{l}{ Case Rep Dermatol 2015;7:183-186 } \\
\hline DOI: 10.1159/000438506 & $\begin{array}{l}\text { ○ 2015 S. Karger AG, Basel } \\
\text { www.karger.com/cde }\end{array}$ \\
\hline
\end{tabular}

Akasaka et al.: Diaminodiphenyl Sulfone-Induced Hemolytic Anemia and Alopecia in a Case of Linear IgA Bullous Dermatosis

\section{Introduction}

Linear immunoglobulin A (IgA) bullous dermatosis (LABD) is an autoimmune mucocutaneous disease characterized by subepidermal blistering induced by IgA autoantibodies against several autoantigens in the basal membranous zone of the skin and mucosal tissue with stratified squamous epithelia. Diaminodiphenyl sulfone (DDS), also known as dapsone, is considered the first-line therapy for LABD. Its efficacy has been well recognized; however, physicians should be aware that DDS can cause several severe side effects. Herein, we report a case of LABD with DDS-induced hemolytic anemia and alopecia, in which iron deficiency anemia (IDA) concealed hemolytic anemia.

\section{Case Report}

A 38-year-old woman was referred to our department with a 3-month history of pruritic skin lesions with tense blisters on her trunk. The skin lesions were resistant to $20 \mathrm{mg} /$ day prednisolone (PSL), administered by the previous dermatologist. The patient had mild IDA. Physical examination revealed multiple rice- to nail-sized erythematous and pigmented patches accompanied by small vesicles and crusts scattered mainly on her trunk (fig. 1a). The individual lesions partially formed a linear and annular arrangement of tiny tense vesicles around reddish eruptions (fig. 1b). The histopathology of a skin biopsy specimen obtained from her chest revealed a subepidermal blister with accumulation of neutrophils and eosinophils (fig. 1c). Direct immunofluorescence of the perilesional skin showed a linear deposition of IgA, but not IgG, at the epidermal basement membrane zone (fig. 1d; http://www.sciencedirect.com/science/article/pii/S0190962210018062 - fig. 2). Indirect immunofluorescence detected circulating IgA autoantibodies binding to both the epidermal and dermal sides of $1 \mathrm{M}$ sodium chloride-split normal human skin (fig. 1e; http://www.sciencedirect.com/science/article/pii/S0190962210018062 - fig. 2), confirming the diagnosis of LABD. An enzyme-linked immunosorbent assay using recombinant protein of the NC16a domain of type XVll collagen showed negative results. No positive results were seen in immunoblotting analyses using human epidermal and dermal extracts $[1,2]$, recombinant NC16a protein [3] and C-terminal domains of type XVII collagen [4], purified human laminin-332 [5] and concentrated HaCaT cell culture supernatant [6].

Based on the diagnosis of LABD, $75 \mathrm{mg} /$ day of oral DDS was added to $20 \mathrm{mg} /$ day of oral PSL. Although the pruritic eruptions dramatically diminished in response to treatment, anemia worsened within 1 month after initiation of oral DDS treatment. The hemoglobin level decreased from 13.2 to $10.6 \mathrm{~g} / \mathrm{dl}$ in normocytic normochromic anemia. Serum iron levels, ferritin and unsaturated iron-binding capacity were within normal ranges. Suspecting hemolytic anemia due to DDS, the DDS dose was tapered to $25 \mathrm{mg} /$ day, and hemolytic anemia changed to IDA, with decreased serum iron and ferritin levels and increased unsaturated iron-binding capacity. The hemoglobin levels recovered to the normal range within 2 weeks of oral iron therapy. After the initiation of DDS therapy, the patient also complained of hair loss, which improved after decreasing the DDS dose. Twenty-five milligrams/day of DDS and $5 \mathrm{mg} /$ day of PSL were sufficient to control the skin lesions. 
Akasaka et al:: Diaminodiphenyl Sulfone-Induced Hemolytic Anemia and Alopecia in a Case of Linear IgA Bullous Dermatosis

\section{Discussion}

DDS is used as the first-line therapy for LABD [7], either as monotherapy [8] or combined with other drugs including corticosteroids [7, 9]. The potential side effects of DDS involve hemolysis, methemoglobinemia, agranulocytosis, distal motor neuropathy, nephritic syndrome, drug-induced hypersensitivity syndrome, cholestatic hepatitis, hypoalbuminemia, pancreatitis, erythema multiformis, toxic epidermal necrosis, and others [10]. In our case, DDS was effective; however, hemolytic anemia and hair loss made the DDS monotherapy difficult. When DDS is used in LABD patients with IDA, hemolytic anemia is concealed by IDA. Hence, it is necessary to carefully and frequently examine the laboratory data to find the signs of DDS-induced hemolytic anemia.

Although there is no previous report describing DDS-induced alopecia, the FDA reported that alopecia accounted for $1.25 \%$ of all side effects of DDS (http://www.ehealthme.com/ds/dapsone/alopecia). A reduced DDS dosage improved the hair loss in our case. Therefore, we speculated that the hair loss was induced by DDS. The exact mechanisms underlying DDS-induced hair loss were not clearly elucidated. Hair loss may also be considered as a systemic lupus erythematosus-like symptom, which is a rare side effect of DDS, even though the patient did not present any other symptoms related to systemic lupus erythematosus. We have to recognize alopecia as a side effect of DDS.

In conclusion, we report a case of LABD with mild hemolytic anemia and hair loss, which were probably caused by DDS. Careful management is necessary in order not to overlook the adverse side effects of DDS when treating LABD patients.

\section{Statement of Ethics}

The authors state that the patients gave their informed consent and that the research complies with all ethical guidelines for human studies.

\section{Disclosure Statement}

The authors declare no conflicts of interest or financial support.

\section{References}

1 Sugi T, Hashimoto T, Hibi T, Nishikawa T: Production of human monoclonal anti-basement membrane zone (BMZ) antibodies from a patient with bullous pemphigoid (BP) by Epstein-Barr virus transformation. Analyses of the heterogeneity of anti-BMZ antibodies in BP sera using them. J Clin Invest 1989;84:10501055.

-2 Ishii N, Yoshida M, Hisamatsu Y, Ishida-Yamamoto A, Nakane H, Iizuka H, Tanaka T, Hashimoto T: Epidermolysis bullosa acquisita sera react with distinct epitopes on the NC1 and NC2 domains of type VII collagen: study using immunoblotting of domain-specific recombinant proteins and postembedding immunoelectron microscopy. Br J Dermatol 2004;150:843-851.

3 Matsumura K, Amagai M, Nishikawa T, Hashimoto T: The majority of bullous pemphigoid and herpes gestationis serum samples react with NC16a domain of the 180-kDa bullous pemphigoid antigen. Arch Dermatol Res 1996;288:507-509.

4 Nie Z, Hashimoto T: IgA antibodies of cicatricial pemphigoid sera specifically react with C-terminus of BP180. J Invest Dermatol 1999;112:254-255.

5 Hisamatsu Y, Nishiyama T, Amano S, Matsui C, Ghohestani R, Hashimoto T: Usefulness of immunoblotting using purified laminin 5 in the diagnosis of anti-laminin 5 cicatricial pemphigoid. J Dermatol Sci 2003;33:113-119. 
Akasaka et al.: Diaminodiphenyl Sulfone-Induced Hemolytic Anemia and Alopecia in a Case of Linear IgA Bullous Dermatosis

6 Ishii N, Ohyama B, Yamaguchi Z, Hashimoto T: IgA autoantibodies against the NC16a domain of BP180 but not 120-kDa LAD-1 detected in a patient with linear IgA disease. Br J Dermatol 2008;158:1151-1153.

7 Jabłońska S: The therapies for linear IgA bullous dermatosis of childhood. Pediatr Dermatol 1999;16:415.

-8 Wojnarowska F: Linear IgA dapsone responsive bullous dermatosis. J R Soc Med 1980;73:371-373.

-9 Jabłońska S, Chorzelski TP, Rosinska D, Maciejowska E: Linear IgA bullous dermatosis of childhood (chronic bullous dermatosis of childhood). Clin Dermatol 1991;9:393-401.

10 Fortuna G, Marinkovich MP: Linear immunoglobulin A bullous dermatosis. Clin Dermatol 2012;30:38-50.
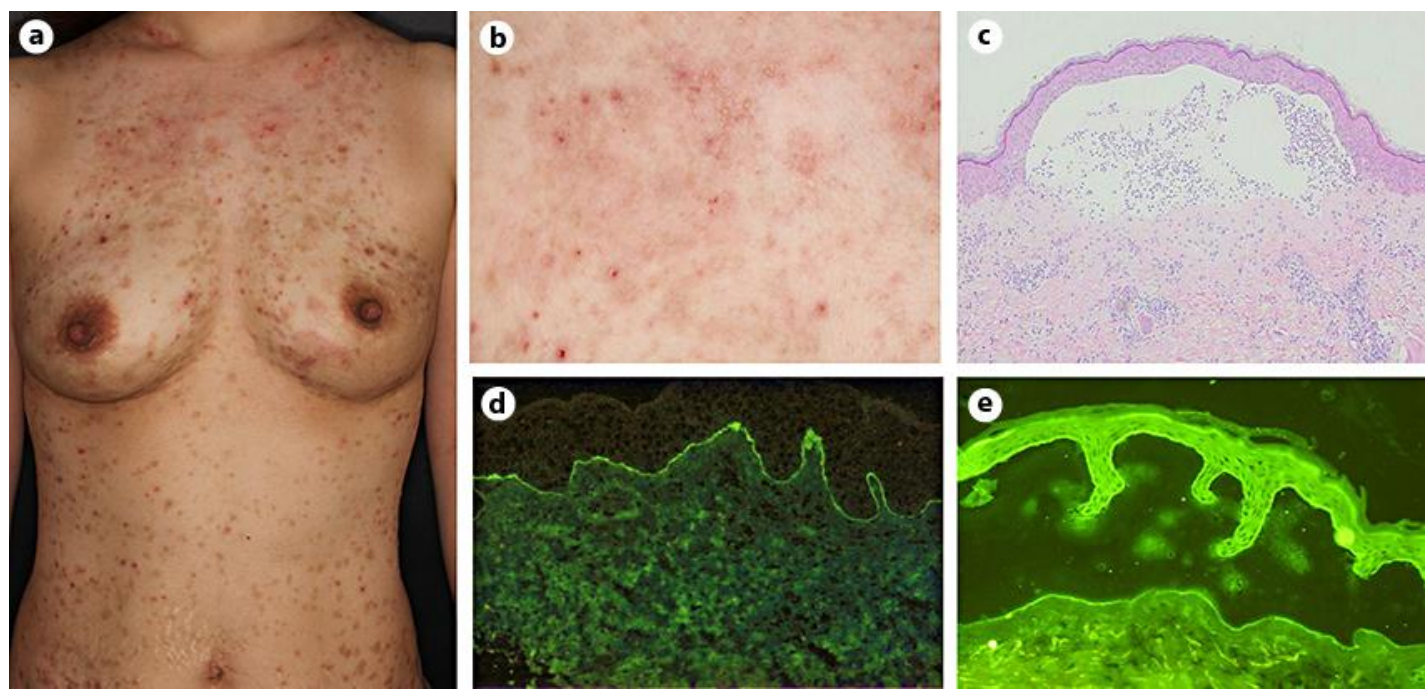

Fig. 1. a, b Clinical manifestation. Skin lesions on the trunk (a), and close-up of the small blisters on the chest (b). c Histopathological findings of the skin biopsy. H\&E. $\times 100$. d, e Direct immunofluorescence for IgA (d), and IgA indirect immunofluorescence of $1 \mathrm{M} \mathrm{NaCl-split} \mathrm{normal} \mathrm{human} \mathrm{skin} \mathrm{(e).}$ 